Empirical modeling of the refractive index for (AIGaln)As lattice matched to InP

This article has been downloaded from IOPscience. Please scroll down to see the full text article.

2010 Semicond. Sci. Technol. 25045018

(http://iopscience.iop.org/0268-1242/25/4/045018)

View the table of contents for this issue, or go to the journal homepage for more

Download details:

IP Address: 129.187.254.46

The article was downloaded on 31/10/2011 at 15:06

Please note that terms and conditions apply. 


\title{
Empirical modeling of the refractive index for (AlGaIn)As lattice matched to InP
}

\author{
C Grasse, G Boehm, M Mueller, T Gruendl, R Meyer and M-C Amann \\ Walter Schottky Institut, Technische Universitaet Muenchen, Am Coulombwall 3, 85748 Garching, \\ Germany \\ E-mail: grasse@wsi.tum.de
}

Received 27 December 2009, in final form 9 February 2010

Published 9 March 2010

Online at stacks.iop.org/SST/25/045018

\begin{abstract}
A composition-dependent empirical interpolation formula for the refractive index of AlGaInAs epilayers lattice matched to InP substrate has been determined by using a reflection spectroscopy technique. The $2 \mu \mathrm{m}$ thick (AlGaIn)As layers have been grown by MBE as well as MOVPE and were characterized by $\mathrm{x}$-ray diffractometry and photoluminescence measurements. The measured data from these etalon structures were fitted by using a Sellmeier equation (single oscillator model) to retrieve the refraction index for wavelengths between 2.5 and $1 \mu \mathrm{m}$. The resulting accurate expression will be very useful for designing optoelectronic devices like VCSELs.
\end{abstract}

(Some figures in this article are in colour only in the electronic version)

\section{Introduction}

The AlGaInAs/InP alloy has become the standard material system for long wavelength laser diodes like vertical-cavity surface-emitting lasers (VCSELs), and high-speed devices usable for 100-G Ethernet have been fabricated [1]. The improved performance compared to GaInAsP-based laser diodes is mainly ascribed to the higher conduction band offset and therefore reduced electron leakage current from active regions [2]. Besides this feature, also the technological advantage of a single group $\mathrm{V}$ system to create easily digital alloy structures has resulted in extremely long wavelength VCSELs by using V-shaped (AlGaIn)As quantum wells grown by MBE [3]. Since the minimization of free carrier losses and the positioning of the tunnel junction, which is needed as current aperture, into a node of the optical field is essential for optimal performance and low threshold currents for such devices, precise knowledge of the corresponding refraction indices is extremely important for the design of the layer structure and doping profile [4].

In this paper, the composition-dependent refractive index of (AlGaIn)As including dispersion is derived by reflection spectroscopy using a new fitting procedure for the whole measured data range, yielding an up to now unmatched accuracy. These data are compared with the reported values from Mondry et al [5] and Nojima et al [6], who used a similar technique. Significant deviations, which are critical for design considerations, could be revealed.

\section{Experiment}

The epitaxy of (AlGaIn)As layers has been carried out with a conventional solid state Varian Mod-Gen II MBE (equipped with valved arsenic and phosphorus cracker cells) and an Aixtron 200/4 MOVPE system (equipped with standard precursors) on iron doped, exactly orientated (0 0101$)$ InP substrates. As growth temperature we used $520^{\circ} \mathrm{C}$ (pyrometer) for MBE grown layers and $650{ }^{\circ} \mathrm{C}$ in the case of MOVPE. The composition of the layers was adjusted by changing the cell temperatures or gasflows, respectively, and not by using the digital alloy technique described in [5]. Characterization of the layers had been performed by double crystal X-ray diffraction (XRD, (004) and (006) reflex) and lowtemperature photoluminescence (PL). The determination of the layer composition has been carried out by combining the PL- and XRD-measurements using the band parameters of [7] and the interpolation scheme of Donati [8].

Table 1 summarizes the grown layers using the nomenclature of a so-called effective quaternary composition $X$ corresponding to a $\left(\mathrm{Al}_{0.48} \mathrm{InAs}\right)_{X}\left(\mathrm{Ga}_{0.47} \mathrm{InAs}\right)_{1-X}$ alloy as introduced in [5]. It should be noted that all layers showed a mirror like surface and exhibit small PL-linewidths (at 
Table 1. Characterization of used $\left(\mathrm{Al}_{0.48} \mathrm{InAs}\right)_{X}\left(\mathrm{Ga}_{0.47} \mathrm{InAs}\right)_{1-X}$.

\begin{tabular}{lllllllll}
\hline $\begin{array}{l}\text { Sample } \\
\text { no }\end{array}$ & Description & $\begin{array}{l}\Delta a / a \\
(\mathrm{rel} .)\end{array}$ & $\begin{array}{l}E_{\mathrm{PL}} \text { at } \\
4.2 \mathrm{~K}^{\mathrm{a}}\end{array}$ & $\begin{array}{l}\mathrm{FWHM} \\
(\mathrm{meV})\end{array}$ & $\begin{array}{l}E_{\mathrm{PL}} \text { at } \\
300 \mathrm{~K}\end{array}$ & $\begin{array}{l}d_{\text {Dektak }} \\
(\mathrm{nm})\end{array}$ & $\begin{array}{l}d_{\text {Sim }} \\
(\mathrm{nm})\end{array}$ & $\begin{array}{l}\mathrm{Al}_{0.48} \mathrm{InAs} \\
X \text { fraction }\end{array}$ \\
\hline 1 & $\mathrm{Al}_{0.48} \mathrm{InAs}$ & $-1.4 \times 10^{-4}$ & 1.516 & 11 & 1.449 & 1945 & 1945 & 1 \\
2 & $\mathrm{Al}_{0.36} \mathrm{GaInAs}$ & $-2.8 \times 10^{-4}$ & 1.341 & 9 & 1.275 & 2013 & 2017 & 0.768 \\
3 & $\mathrm{Al}_{0.24} \mathrm{GaInAs}$ & $3.6 \times 10^{-4}$ & 1.144 & 7 & 1.077 & 1975 & 1969 & 0.502 \\
4 & $\mathrm{Al}_{0.12} \mathrm{GaInAs}$ & $2.8 \times 10^{-6}$ & 0.984 & 8 & 0.921 & 2029 & 2027 & 0.268 \\
5 & $\mathrm{Al}_{0.12} \mathrm{GaInAs}$ & $-2.4 \times 10^{-4}$ & 0.979 & 4 & 0.903 & 2248 & 2241 & 0.235 \\
6 & $\mathrm{Ga}_{0.47} \mathrm{InAs}$ & $2.2 \times 10^{-4}$ & 0.799 & 5 & 0.737 & 2008 & 2003 & 0 \\
7 & $\mathrm{Al}_{0.85} \mathrm{GaAs}$ & $1.3 \times 10^{-3}$ & - & - & - & - & 1938 & $0.883^{\mathrm{a}}$ \\
8 & $\mathrm{Al}_{0.14} \mathrm{GaAs}$ & $2.2 \times 10^{-4}$ & - & - & - & - & 2050 & $0.136^{\mathrm{a}}$ \\
\hline
\end{tabular}

${ }^{\mathrm{a}} X=x_{\mathrm{Al}}$.

4.2 K) indicating a perfect material quality. To achieve a comparison between our findings in this rather new material system, AlGaAs layers have also been grown and investigated in the same way. For composition determination the more accurate ternary lattice constant after [9] has been used in this case, since deviations from the Vegard law have been reported for this alloy.

To measure the refractive index we used a reflectance technique already described in [5]. It is based on a Fabry-Pèrot etalon structure consisting of an epitaxial layer of unknown refractive index $n_{L}$ on a substrate. The spectra have been measured with a Bruker Vertex V70 FTIR spectrometer. An extended InGaAs-diode served as a detector. The measured spotsize had a radius of $1 \mathrm{~mm}$, which excludes homogeneity issues.

For the reflection coefficient $r$ of this structure, where $r_{0}$ is the field reflectivity at the epitaxial layer/air interface and $r_{1}$ is the reflection at the epitaxial layer/substrate interface, we obtain

$$
r=\frac{r_{0}+r_{1} \cdot \mathrm{e}^{-2 \mathrm{j} d k}}{1+r_{0} \cdot r_{1} \cdot \mathrm{e}^{-2 \mathrm{j} d k}},
$$

where $d$ is the layer thickness and $k$ is the wavevektor inside the grown layer. Given that only top-side-polished substrates have been applied, the backside reflection can be neglected.

Since the reflectivity $R$ is simply given by the squared absolute value of the field reflection, the measured reflection signal can be simulated by knowing $d$ as well as the refraction index of the layer and the substrate $\left(n_{\text {Air }}=\right.$ 1). However, as shown in figure 1, measured data are affected by detector sensitivity, absorption lines and the excitation spectrum. Therefore, it is more useful to fit the ratio $R / R_{i}$ to eliminate these uncertainties [5], where $R_{i}$ is the reflectivity of the corresponding substrate against air. Figure 2 shows the resulting normalized reflection spectra (straight line) for AlGaInAs/InP (upper line) and an AlGaAs/GaAs sample. Since all layers are undoped and only measured values below the layer bandgap (at least $100 \mathrm{meV}$ to avoid the Urbach tail) have been utilized, absorption could be disregarded. Therefore, both normalized spectra oscillate around unity, which means that the fringe minima (in the case of AlGaInAs) and maxima (in the case of AlGaAs) are equal to the substrate/air reflection, as it is theoretically expected when the optical thickness of the layer corresponds to an even integer number of a quarter wavelength [5].

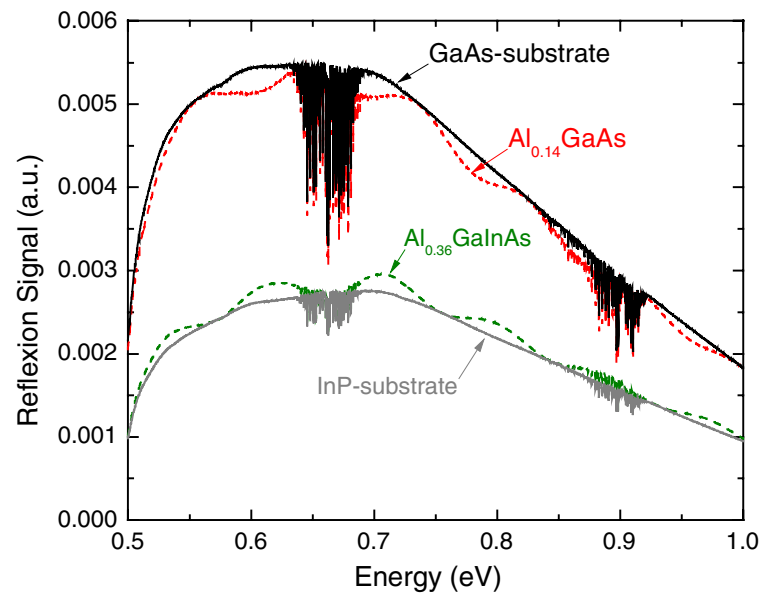

Figure 1. Measured reflection spectra from Fabry-Perot etalons are illustrated. Although the spectrometer and probe chamber are purged by nitrogen, $\mathrm{H}_{2} \mathrm{O}$ absorption lines in the spectra could not be avoided.

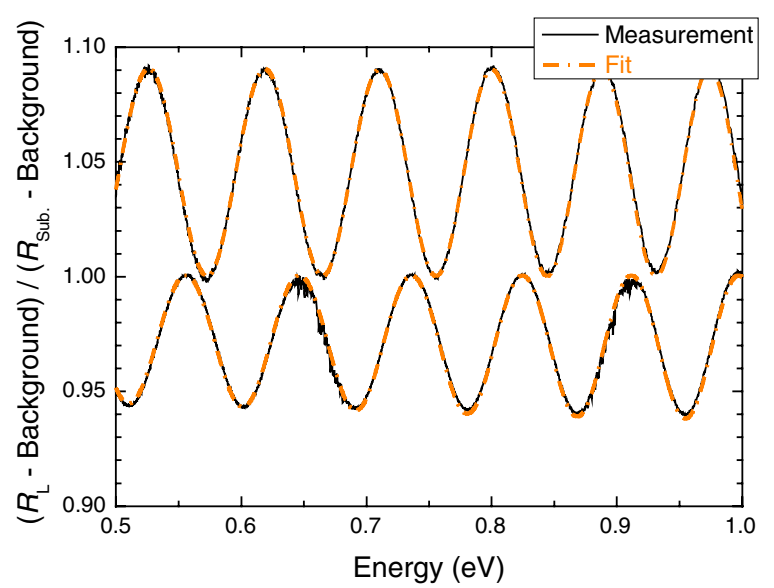

Figure 2. The normalized etalon reflection (straight lines) extracted from the measurements shown in figure 1 are illustrated. The resulting fits are indicated as dashed lines.

To analyze the normalized measurement we need the refractive index data of the substrate, which we have taken from Pettit and Turner for the refraction index of InP [10], whose accuracy have been proven [11, 12], whereas in the case of GaAs we used the formula from Afromowitz [13], also confirmed by Gehrsitz et al [14]. Therefore, only the refractive 
index and the thickness of the layer were released as parameters by using a least square error fit method. The unknown refractive index of the AlGaInAs epilayer was estimated by using a single oscillator Sellmeier equation:

$$
n_{\text {Layer }}(\lambda)=\sqrt{A+\frac{B \cdot \lambda^{2}}{\lambda^{2}-C^{2}}}
$$

where $A, B, C$ are the Sellmeier parameters and $\lambda$ is the wavelength in $\mu \mathrm{m}$. To prove the simulations the thickness of the (AlGaIn)As samples was also measured by using a Veeco Dektak surface profiler and selective chemical wet etching. The standard deviations of these measurements add up to $0.2-0.3 \%$ and they are also listed in table 1 . Comparing the measured thickness with simulated values leads to the new finding that this technique is also very useful and accurate as non-destructive thickness determination, which can also be applied to other material systems.

\section{Results and discussion}

The AlGaInAs fitting data of samples 1-4 and 6 have been used to create a third-order interpolation matrix with respect to the composition $X$ for the refraction index of this material system lattice matched to InP:

$$
\begin{aligned}
\left(\begin{array}{l}
A \\
B \\
C
\end{array}\right) & =\left(\begin{array}{llll}
10.761 & -2.2617 & -5.3678 & 5.4943 \\
0.9485 & 0.2369 & 5.2374 & -5.1947 \\
1.5986 & -3.0266 & 2.3326 & -0.3629
\end{array}\right) \\
& \cdot\left(\begin{array}{c}
1 \\
X \\
X^{2} \\
X^{3}
\end{array}\right) .
\end{aligned}
$$

A maximum error between interpolation and direct fit of $2 \times 10^{-7}$ shows excellent accuracy. Figure 3 compares the index dispersion of (AlGaIn)As with various 'effective' aluminum fractions $X$. Interestingly, our data agrees well with Mondry et al [5] in the case of AlInAs, whereas for GaInAs Nojima's [6] findings are proven. This means that we have an average variance of $0.2 \%$ and $1.2 \%$ between our data and [5] and [6] respectively for $X=1$ (AlInAs). Decreasing the aluminum content results into less agreement with Mondry et al [5], but better accordance with Nojima [6], so that for $X=0$ (GaInAs) the average deviation becomes $1.5 \%$ and $0.2 \%$ respectively. However, these discrepancies can be explained by the fact that Mondry et al have applied their fitting technique only to AlGaInAs layers with a minimum $X$ fraction of 0.28 and took the dispersion data for GaInAs from earlier work. Also the fitting procedure just included the reflectivity maxima and minima and not the whole spectrum as in our case, which explains the differences of the refractive index dispersion. Nojima on the other hand did not detail the material quality of the grown samples and reported a varying refraction index for different growth methods. In the present work on the other hand the MOVPE grown sample (no 5) showed good agreement $(<0.1 \%)$ between direct fit and interpolation, therefore our findings are independent of the growth technique as expected. Also the thickness measurements listed in table 1 agree with a maximum deviation of $\pm 0.3 \%$ in the

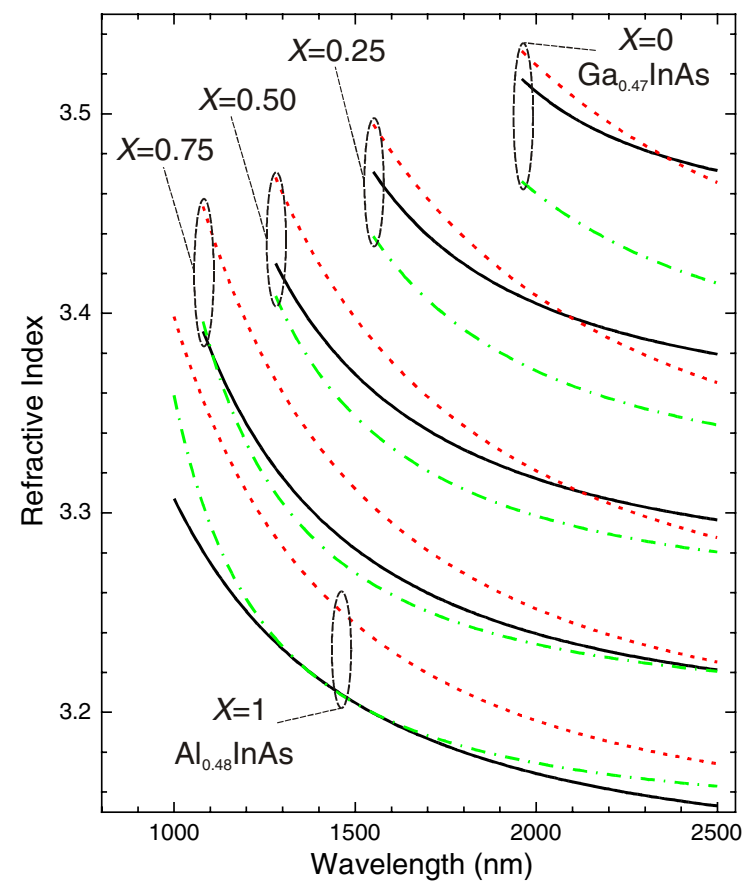

Figure 3. A comparison of refractive index dispersion data for various effective $X$ fractions of $\left(\mathrm{Al}_{0.48} \mathrm{InAs}\right)_{X}\left(\mathrm{Ga}_{0.47} \mathrm{InAs}\right)_{1-X}$ is illustrated. The dashed-dotted lines shows the fit after [5], the dashed line corresponds to [6], whereas the straight line represents our data using formula (3).

simulated layer thickness. Furthermore, comparing fitting data of the AlGaAs layers with expected values from the literature $[13,14]$ yields a maximum deviance of $\pm 0.3 \%$, which is mainly attributed to the usual uncertainties in the composition analysis of around $1 \%$ (absolute) with respect to $X$ [14]. We could therefore exactly reproduce the existing refraction index data of the well-explored AlGaAs alloy, which strengthen our results for the AlGaInAs material system.

Finally it should be noted that we measured for the $\mathrm{Al}_{0.48}$ InAs and $\mathrm{Ga}_{0.47}$ InAs alloy the largest refractive index difference compared to earlier reports (see figure 3 ). Since the bandwidth of an epitaxial DBR consisting of these alloys is defined by this difference, simulations of grown mirrors gave further evidence for our data. Furthermore, it is obvious that with the data of [5] and [6] it is not possible to grow such a DBR and hit the desired Bragg wavelength, which also influences the laser wavelength. Deviations up to $1.5 \%$ (at $2 \mu \mathrm{m}$ ) of the refraction index data correspond to a wavelength shift of around $30 \mathrm{~nm}$, which is not acceptable for applications such as gas sensing [15]. Therefore, to promote new application areas for such VCSEL devices, which require fine wavelength control like gas sensing, accurate refraction index data are absolutely necessary.

In conclusion, we have calculated an empirical composition-dependent third-order interpolation matrix for the dispersion of the refractive index of the (AlGaIn)As material system. The calculation is based on normalized reflection spectra of five Fabry-Pèrot etalon structures with varying aluminum composition grown by MBE. Our findings have been proven by a further sample grown by MOVPE, independent thickness measurements and a comparison 
between measured results of AlGaAs samples with literature data. The perfect agreement between the simulated and measured sample thickness indicates that this reflection technique can also be used as nondestructive thickness determination. At last, since our data argue for a higher refractive index difference between $\mathrm{Al}_{0.48}$ InAs and $\mathrm{Ga}_{0.47} \mathrm{InAs}$ than that in former reports, an essential improvement for the design of (AlGaIn)As-based optoelectronic devices such as VCSELs can be expected.

\section{Acknowledgments}

Financial support by the European Union in the framework of the project SUBTUNE (project no 224259) and the Excellence Cluster 'Nanosystems Initiative Munich (NIM)' is gratefully acknowledged.

\section{References}

[1] Mueller M, Hofman W, Bohm G and Amann M-C 2009 Short-cavity long-wavelength VCSELs with modulation bandwidths in excess of $15 \mathrm{GHz}$ IEEE Photonics Technol. Lett. 21 1615-7

[2] Piprek J, White J K and SpringThorpe A J 2002 What limits the maximum output power of long-wavelength AlGaInAs/InP laser diodes? IEEE J. Quantum Electron. 38 1253-9

[3] Boehm G, Grau M, Dier O, Windhorn K, Roenneberg E, Rosskopf J, Shau R, Meyer R, Ortsiefer M and Amann M-C 2007 Growth of InAs-containing quantum wells for InP-based VCSELs emitting at $2.3 \mu \mathrm{m} \mathrm{J}$. Cryst. Growth 301-302 941-4

[4] Feezell D, Buell D A, Lofgreen D, Mehta M and Coldren L A 2006 Optical design of InAlGaAs low-loss tunnel-junction apertures for long-wavelength vertical-cavity lasers IEEE J. Quantum Electron. 42 494-9

[5] Mondry M J, Babic D I, Bowers J E and Coldren L A 1992 Refractive indexes of (Al, Ga, In)As epilayers on InP for optoelectronic applications IEEE Photonics Technol. Lett. $4627-30$

[6] Nojima S and Asahi H 1988 Refractive index of InGaAs/InAlAs multiquantum-well layers grown by molecular-beam epitaxy J. Appl. Phys. 63 479-83

[7] Vurgaftman I, Meyer J R and Ram-Mohan L R 2001 Band parameter for III-V compound semiconductors and their alloys J. Appl. Phys. 89 5815-73

[8] Donati G P, Kaspi R and Malloy K J 2003 Interpolating semiconductor alloy parameters: application to quaternary III-V band gaps J. Appl. Phys. 94 5814-9

[9] Gehrsitz S, Sigg H, Herres N, Köhler K, Bachem K and Reinhart F K 1999 Compositional dependence of the elastic constants and the lattice parameter of $\mathrm{Al}_{x} \mathrm{Ga}_{1-x} \mathrm{As}$ Phys. Rev. B 6011601

[10] Pettit G D and Turner W J 1965 Refractive index of InP J. Appl. Phys. 362081

[11] Broberg B and Lindgren S 1984 Refractive index of $\mathrm{In}_{1-x} \mathrm{Ga}_{x} \mathrm{As}_{y} \mathrm{P}_{1-y}$ layers and $\mathrm{InP}$ in the transparent wavelength region J. Appl. Phys. 55 3376-81

[12] Henry C H, Johnson L F, Logan R A and Clark D P 1985 Determination of the refractive index of InGaAsP epitaxial layers by mode line luminescence spectroscopy IEEE $J$. Quantum Electron. 21 1887-92

[13] Afromowitz M A 1974 Refractive index of $\mathrm{Ga}_{1-x} \mathrm{Al}_{x}$ As Solid State Commun. 15 59-63

[14] Gehrsitz S, Reinhart F K, Gourgon C, Herres N, Vonlanthen A and Sigg $\mathrm{H} 2000$ The refractive index of $\mathrm{Al}_{x} \mathrm{Ga}_{1-x}$ As below the band gap: Accurate determination and empirical modeling J. Appl. Phys. 87 7825-37

[15] Werle P 1998 A review of recent advances in semiconductor laser based gas monitors Spectrochim. Acta A 54 197-236 